\title{
Hamiltonian-Laceability of Star Graphs
}

\author{
Sun-yuan Hsieh \\ Dept. of Computer Science \& Info. Engg. \\ National Taiwan University, Taiwan \\ e-mail: d3506013@csie.ntu.edu.tw
}

\author{
Gen-Huey Chen \\ Dept. of Computer Science \& Info. Engg. \\ National Taiwan University, Taiwan \\ e-mail: ghchen@csie.ntu.edu.tw
}

\author{
Chin-Wen Ho \\ Dept. of Computer Science \& Info. Engg. \\ National Central University, Taiwan \\ e-mail: hocw@csie.ncu.edu.tw
}

\begin{abstract}
Suppose $G$ is a bipartite graph with two partite sets of equal size. $G$ is said to be strongly hamiltonianlaceable if there is a hamiltonian path between every two vertices that belong to different partite sets, and there is a path of (maximal) length $N-2$ between every two vertices that belong to the same partite set, where $N$ is the order of $G$. The star graph is known to be bipartite. In this paper, we show that the $n$-dimensional star graph, where $n \geq 4$ is strongly hamiltonian-laceable.
\end{abstract}

\section{Introduction}

Usually when the hamiltonicity of a graph $G$ is concerned, it is investigated whether $G$ is hamiltonian or hamiltonian-connected. A cycle (path) in $G$ is called a hamiltonian cycle (path) if it contains every vertex of $G$ exactly once. $G$ is said to be hamiltonian if it contains a hamiltonian cycle, and hamiltonianconnected if there exists a hamiltonian path between every two vertices of $G$. Since a bipartite graph is not hamiltonian-connected, Wong [5] has introduced the concept of hamiltonian-laceability for the class of bipartite graphs. A bipartite graph $G=(V 1, V 2, E)$ with $|V 1|=|V 2|$ is hamiltonian-laceable if there is a hamiltonian path between every vertex of $V_{1}$ and every vertex of $V_{2}$, where $V_{1}$ and $V_{2}$ are the two partite sets of $G$. We note that any path between two vertices of the same partite set has length at most $|V 1|+|V 2|-2$.

It is meaningful to extend the concept of hamiltonian-laceability so that the lengths of the paths between two vertices of the same partite set are specified and the edge faults are considered. We say that a hamiltonian-laceable graph $G=(V 1, V 2, E)$ is strongly if $G$ additionally owns the property that there is a path of length $|V 1|+|V 2|-2$ between every two vertices of the same partite set. Further, $G$ is $k$ edge fault-tolerant strongly hamiltonian-laceable if it remains strongly hamiltonian-laceable after removing at most $k$ edges. In other words, there is a longest path between every two vertices of a $k$ edge fault-tolerant strongly hamiltonian-laceable graph $G$, even if at most $k$ edges of $G$ are removed. The longest path has length
$|V 1|+|V 2|-1$ if the two vertices belong to different partite sets, and $|V 1|+|V 2|-2$ if the two vertices belong to the same partite set.

The star graph [1], which belongs to the class of Cayley graphs, has been recognized as an attractive alternative to the hypercube. It possesses many nice topological properties, e.g., recursiveness, vertex and edge symmetry, maximal fault tolerance, sublogarithmic degree and diameter, and strong resilience [1] [2], which are desirable when we are building an interconnection topology for parallel and distributed systems. In [3], Jwo, Lakshmivarahan, and Dhall have shown that the star graph is bipartite. Besides, its two partite sets have equal size. In this paper we show that the $n$-dimensional star graph is strongly hamiltonianlaceable when $n \geq 4$.

\section{Prelimiaries}

The $n$-dimensional star graph, denoted by $S_{n}$, is defined as follows.

Definition 1 The vertex set of $S_{n}$ is denoted by $\left\{a_{1} a_{2} \ldots a_{n} \mid a_{1} a_{2} \ldots a_{n}\right.$ is a permutation of $\left.\{1,2, \ldots, n\}\right\}$. Vertex adjacency is defined as follows: $a_{1} a_{2} \ldots a_{n}$ is adjacent to $a_{i} a_{2} \ldots a_{i-1} a_{1} a_{i+1} \ldots a_{n}$ for all $2 \leq i \leq n$. The vertices of $S_{n}$ are $n$ ! permutations of $\{1,2, \ldots, n\}$, and there is an edge between two vertices of $S_{n}$ if and only if they can be obtained from each other by swapping the leftmost number with one of the other $n-1$ numbers. For convenience we refer to the position of $a_{i}$ in $a_{1} a_{2} \ldots a_{n}$ as the ith dimension, and $\left(a_{1} a_{2} \ldots a_{n}, a_{i} a_{2} \ldots a_{i-1} a_{1} a_{i+1} \ldots a_{n}\right)$ as the ithdimensional edge.

Definition 2 There are embedded $S_{r}$ 's contained in $S_{n}$, where $1 \leq r \leq n$. An embedded $S_{r}$ can be conveniently represented by $\left\langle s_{1} s_{2} \ldots s_{n}\right\rangle_{r}$, where $s_{1}=*$, $s_{i} \in\{*, 1,2, \ldots, n\}$ for all $2 \leq i \leq n$, and exactly $r$ of $s_{1}, s_{2}, \ldots, s_{n}$ are * (* denotes a "don't care" symbol).

Definition $3 A n$ i-partition on $\left\langle s_{1} s_{2} \ldots s_{n}\right\rangle_{r}$ partitions $\left\langle s_{1} s_{2} \ldots s_{n}\right\rangle_{r}$ into $r$ embedded $S_{r-1}^{\prime} s$, denoted by $<s_{1} s_{2} \ldots s_{i-1} q s_{i+1} \ldots s_{n}>_{r-1}$, where $2 \leq i \leq n$, $s_{i}=*$, and $q \in\{1,2, \ldots, n\}-\left\{s_{1}, s_{2}, \ldots, s_{n}\right\}$. 
Definition $4 A n$

$\left(i_{1}, i_{2}, \ldots, i_{m}\right)$ partition on $\left\langle s_{1} s_{2} \ldots s_{n}\right\rangle_{r}$ performs an $i_{1}-$ partition, an $i_{2}$-partition, ..., an $i_{m}$-partition, sequentially, on $\left.<s_{1} s_{2} \ldots s_{n}\right\rangle_{r}$, where $i_{1} i_{2} \ldots i_{m}$ is a permutation of $m$ elements from $\{2,3, \ldots, n\}$.

Definition 5 Two embedded $S_{r}$ 's $\left\langle s_{1} s_{2} \ldots s_{n}\right\rangle_{r}$ and $\left\langle t_{1} t_{2} \ldots t_{n}\right\rangle_{r}$ are said to be adjacent if $s_{j} \neq *, t_{j} \neq *$, and $s_{j} \neq t_{j}$ for some $2 \leq j \leq n$, and $s_{i}=t_{i}$ for all $1 \leq i \leq n$ and $i \neq j$. Moreover, the position $j$ is deñoted by dif $\left.\left.\left(<s_{1} s_{2} \ldots s_{n}\right\rangle_{r},<t_{1} t_{2} \ldots t_{n}\right\rangle_{r}\right)$.

Definition 6 Let $A_{1}, A_{2}, \ldots, A_{n(n-1)(n-2) \cdots(r+1)}$ represent those embedded $S_{r}$ 's that are obtained by executing an $\left(i_{1}, i_{2}, \ldots, i_{n-r}\right)$-partition on $S_{n}$, where $1 \leq r \leq n-1$. They form an $r$-path, denoted by $P_{r}=\left[\overline{A_{1}}, A_{2}, \ldots, A_{n(n-1)(n-2) \cdots(r+1)}\right]$, if $A_{i}$ is adjacent to $A_{i+1}$ for all $1 \leq i \leq n(n-1)(n-2) \cdots(r+1)-1$. Each vertex of $P_{r}$ i.e., $A_{i}$, is called an r-vertex, and each edge of $P_{r}$, i.e., $\left(A_{i}, A_{i+1}\right)$, is called an $r$-edge.

Definition $7 A n$

$i$-partition on $P_{r}=\left[A_{1}, A_{2}, \ldots, A_{n(n-1)(n-2) \cdots(r+1)}\right]$ performs an $i$-partition on $A_{1}, A_{2}, \ldots, A_{n(n-1)(n-2) \cdots(r+1)}$, respectively, where $2 \leq i \leq n$ and $r \geq 2$. After an $i$ partition, each $A_{j}$ is partitioned into $r(r-1)$-vertices, where $1 \leq j \leq n(n-1)(n-2) \cdots(r+1)$. Since every two of the $r(r-1)$-vertices are joined with an $(r-1)$ edge, each $A_{j}$ can be viewed as a complete graph of $r$ $(r-1)$-vertices. Throughout this paper, we refer to the complete graph as $K_{r}^{r-1}$. We note that each vertex of $K_{r}^{r-1}$ is an $(r-1)$-vertex and each edge of $K_{r}^{r-1}$ is an $(r-1)$-edge.

\section{Hamiltonian-Laceability of Star Graphs}

In this section we show $S_{n}$ with $n \geq 4$ is strongly hamiltonian-laceable.

Lemma 3.1 Suppose $U=\left\langle u_{1} u_{2} \ldots u_{n}\right\rangle_{r}, V=$ $\left.<v_{1} v_{2} \ldots v_{n}\right\rangle_{r}$, and $W=\left\langle w_{1} w_{2} \ldots w_{n}\right\rangle_{r}$ are arbitrary three consecutive r-vertices in a $P_{r}$, where $r \geq 2$. Let $p=\operatorname{dif}(U, V)$ and $q=\operatorname{dif}(V, W)$. If $u_{p} \neq w_{q}$, then after executing a partition on the $P_{r}$ each $(r-1)$ vertex of $V$ is connected to $U$ or $W$.

Proof: Without loss of generality, we assume that a $j$-partition is executed on the $P_{r}$, where $2 \leq j \leq n$. Hence, $u_{j}=v_{j}=w_{j}=*$. Since $p=\operatorname{dif}(U, V) \neq 1$ and $q=\operatorname{dif}(V, W) \neq 1$, we have $u_{p} \neq v_{p}, v_{q} \neq w_{q}$, $u_{i}=v_{i}$ for all $1 \leq i \leq n$ and $i \neq p$, and $v_{i}=w_{i}$ for all $1 \leq i \leq n$ and $i \neq q$. Suppose conversely $u_{p} \neq w_{q}$ and there exists an $(r-1)$-vertex, say $V_{1}=\left\langle v_{1} v_{2} \ldots v_{j-1} z v_{j+1} \ldots v_{n}\right\rangle_{r-1}$, of $V$ which is not connected to either of $U$ and $W$. Thus, $z=u_{p}$, for otherwise $V_{1}$ is adjacent to some $(r-1)$-vertex of $U$. Similarly, $z=w_{q}$. This implies $u_{p}=w_{q}$, which contradicts our assumption.

Q.E.D.

Lemma 3.2 Suppose $u$ and $v$ are arbitrary two distinct vertices of $S_{n}$ with $n \geq 4$. There exists a $P_{n-1}$ whose first $(n-1)$-vertex contains $u$ and whose last $(n-1)$-vertex contains $v$.
Proof: Suppose $u=u_{1} u_{2} \ldots u_{n}$ and $v=v_{1} v_{2} \ldots v_{n}$. Without loss of generality, we assume $u_{j} \neq v_{j}$ for some $2 \leq j \leq n$. After a $j$-partition, $S_{n}$ is partitioned into $n(n-1)$-vertices, which form a $K_{n}^{n-1}$. Clearly, $u$ and $v$ belong to two different vertices, say $U$ and $V$, of the $K_{n}^{n-1}$. The desired $P_{n-1}$ can be constructed as a hamiltonian path from $U$ to $V$ in the $K_{n}^{n-1}$. Q.E.D.

In the rest of this paper, we suppose $u$ and $v$ are the beginning vertex and the ending vertex, respectively, of a path. We call an $r$-vertex the beginning $r$-vertex (ending $r$-vertex) if it contains $u(v)$. Besides, a path from $U$ to $V$ is abbreviated to a $U-V$ path.

Lemma 3.3 $A P_{r-1}$ whose first $(r-1)$-vertex is the beginning $(r-1)$-vertex and whose last $(r-1)$-vertex is the ending $(r-1)$-vertex can be obtained from a $P_{r}$ whose first $r$-vertex is the beginning $r$-vertex and whose last $r$-vertex is the ending r-vertex, where $4 \leq$ $r \leq n-1$ and $n \geq 5$.

Proof: $\quad$ Suppose $\quad P_{r} \quad=$ $\left[A_{1}, A_{2}, \ldots, A_{n(n-1)(n-2) \cdots(r+1)}\right]$, where $A_{1}$ is the begin ning $r$-vertex and $A_{n(n-1)(n-2) \cdots(r+1)}$ is the ending $r$ vertex. After executing a partition on the $P_{r}$, each $A_{i}$ forms a $K_{r}^{r-1}$, where $1 \leq i \leq n(n-1)(n-2) \cdots(r+1)$. Since each $A_{i}$ contairs at least three $(r-1)$-vertices, we can select two distinct $(r-1)$-vertices, say $X_{i}$ and $Y_{i}$, from each $A_{i}$ so that $X_{1}$ is the beginning $(r-1)$ vertex, $Y_{n(n-1)(n-2) \cdots(r+1)}$ is the ending $(r-1)$-vertex, and for $2 \leq j \leq n(n-1)(n-2) \cdots(r+1)-1$, $X_{j}$ and $Y_{j}$ are adjacent to $Y_{j-1}$ and $X_{j+1}$, respectively. Since there exists a hamiltonian $X_{i}-Y_{i}$ path in the $K_{r}^{r-1}$ formed by $A_{i}$, the desired $P_{r-1}$ can be obtained by concatenating all the hamiltonian paths interleaved with $(r-1)$-edges $\left(Y_{1}, X_{2}\right),\left(Y_{2}, X_{3}\right), \ldots$ $\left(Y_{n(n-1)(n-2) \cdots(r+1)-1}, X_{n(n-1)(n-2) \cdots(r+1)}\right)$ Q.E.D.

In the rest of this paper, $X_{i}$ and $Y_{i}$ as specified above are referred to as the entry $(r-1)$-vertex and the exit $(r-1)$-vertex of $A_{i}$, respectively.

Lemma 3.4 $A P_{5}$ whose first 5-vertex is the beginning 5-vertex and whose last 5-vertex is the ending 5-vertex can be obtained in $S_{n}$ with $n>5$.

A $P_{r}=\left[A_{1}, A_{2}, \ldots, A_{n(n-1)(n-2) \cdots(r+1)}\right]$ in $S_{n}$, where $2 \leq r \leq n-1$, is said to be good if it satisfies the following three conditions.

(Cond. 1) $A_{1}$ and $A_{n(n-1)(n-2) \cdots(r+1)}$ are the beginning and ending $r$-vertices, respectively.

(Cond. 2) For arbitrary three consecutive $r$-vertices $X=\left\langle x_{1} x_{2} \ldots x_{n}\right\rangle_{r}, Y=\left\langle y_{1} y_{2} \ldots y_{n}\right\rangle_{r}$, and $Z=$

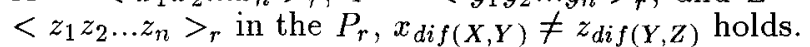
(Cond. 3) After executing a $k$-partition on the $P_{r}$ for some $2 \leq k \leq n$, the beginning (ending) $(r-1)$ vertex in $A_{1}^{-}\left(A_{n(n-1)(n-2) \cdots(r+1)}\right)$ is not connected to $A_{2}\left(A_{n(n-1)(n-2) \cdots(r+1)-1}\right)$.

In the rest of this section we show that a good $P_{3}$ can be obtained in $S_{n}$. Given arbitrary two vertices of $S_{n}$, a longest path between them can be constructed from a $\operatorname{good} P_{3}$. 
Lemma $3.5 A$ good $P_{4}$ can be obtained from a $P_{5}$ whose frrst 5-vertex is the beginning 5-vertex and whose last 5-vertex is the ending 5-vertex.

Proof: We suppose $P_{5}=\left[A_{1}, A_{2}, \ldots, A_{n(n-1)(n-2) \cdots 6}\right]$, where $A_{1}$ and $A_{n(n-1)(n-2) \cdots 6}$ are the beginning and ending 5 -vertices. Without loss of generality, we assume that the $P_{5}$ is obtained from $S_{n}$ by executing an $\left(a_{1}, a_{2}, \ldots, a_{n-5}\right)$-partition, where $a_{1} a_{2} \ldots a_{n-5}$ is an arrangement out of $\{2,3, \ldots, n\}$. Let $j \in\{2,3, \ldots, n\}-$ $\left\{a_{1}, a_{2}, \ldots, a_{n-5}\right\}$. First, a $j$-partition is executed on the $P_{5}$, and so each $A_{i}$ forms a $K_{5}^{4}$, where $1 \leq i \leq$ $n(n-1)(n-2) \cdots 6$. In the rest of the proof we construct a good $P_{4}$ from the $P_{5}$ by establishing a hamiltonian path for each $K_{5}^{4}$.

Suppose $u=u_{1} u_{2} \ldots u_{n}$ and $v=v_{1} v_{2} \ldots v_{n}$ are the beginning and ending vertices, respectively. A hamiltonian path for the $K_{5}^{4}$ formed by $A_{1}$ can be established as follows. Let $X_{1}=\left\langle x_{1} x_{2} \ldots x_{n}\right\rangle_{4}$ be the beginning 4 -vertex (in $A_{1}$ ), $T$ be the 4 -vertex of $A_{1}$ that is not connected to $A_{2}$, and $W=\left\langle w_{1} w_{2} \ldots w_{n}\right\rangle_{4}$ be a 4-vertex of $A_{1}$ which is different from $X_{1}$ and has $w_{j}=u_{k}$ for some $k \in\{2,3, \ldots, n\}-\left\{j, a_{1}, a_{2}, \ldots, a_{n-5}\right\}$. Since there are four 4-edges between $A_{1}$ and $A_{2}$, there exists a 4-vertex $Y_{1} \notin\left\{X_{1}, W\right\}$ which is connected to $A_{2}$. If $X_{1}=T$ or $\left(X_{1} \neq T\right.$ and $\left.T=W\right)$, a hamiltonian $X_{1}-Y_{1}$ path can be established as $\left(X_{1}, W\right)+P\left[W, Y_{1}\right]$, where $P\left[W, Y_{1}\right]$ denotes a $W-Y_{1}$ path passing all the vertices of the $K_{5}^{4}$ but $X_{1}$ exactly once. Otherwise, if $X_{1} \neq T$ and $T \neq W$, a hamiltonian $X_{1}-Y_{1}$ path can be established as $\left(X_{1}, W\right)+$ $(W, T)+P\left[T, Y_{1}\right]$, where $P\left[T, Y_{1}\right]$ denotes a $T-Y_{1}$ path passing all the vertices of the $K_{5}^{4}$ but $X_{1}$ and $W$ exactly once. Then we continue to establish a hamiltonian path for the $K_{5}^{4}$ formed by $A_{n(n-1)(n-2) \cdots 6}$. The construction of the hamiltonian path is similar to the situation of $A_{1}$. Let $Y_{n(n-1)(n-2) \cdots 6}$ be the ending 4-vertex (in $A_{n(n-1)(n-2) \cdots 6}, C$ be the 4-vertex of $A_{n(n-1)(n-2) \cdots 6}$ that is not connected to $A_{n(n-1)(n-2) \cdots 6-1}$, and $D=\left\langle d_{1} d_{2} \ldots d_{n}\right\rangle_{4}$ be the 4-vertex of $A_{n(n-1)(n-2) \cdots 6}$ that is different from $Y_{n(n-1)(n-2) \cdots 6}$ and has $d_{j}=v_{k}$ (here, $k$ is identical with that $k$ appearing in the situation of $\left.A_{1}\right)$. There exists a vertex $X_{n(n-1)(n-2) \cdots 6} \notin\left\{D, Y_{n(n-1)(n-2) \cdots 6}\right\}$ which is connected to $A_{n(n-1)(n-2) \cdots 6-1}$. If $Y_{n(n-1)(n-2) \cdots 6}=C$ or $Y_{n(n-1)(n-2) \cdots 6} \neq C$ and $C=$ $D)$, a hamiltonian $X_{n(n-1)(n-2) \cdots 6}-Y_{n(n-1)(n-2) \cdots 6}$ path can be established as $P\left[X_{n(n-1)(n-2) \cdots 6}, D\right]+$ $\left(D, Y_{n(n-1)(n-2) \cdots 6}\right)$, where $P\left[X_{n(n-1)(n-2) \cdots 6}, D\right]$ denotes an $X_{n(n-1)(n-2) \cdots 6}-D$ path passing all the vertices of the $K_{5}^{4}$ but $Y_{n(n-1)(n-2) \cdots 6}$ exactly once. Otherwise, if $Y_{n(n-1)(n-2) \cdots 6} \neq C$ and $C \neq D$, a hamiltonian $X_{n(n-1)(n-2) \cdots 6}-Y_{n(n-1)(n-2) \cdots 6}$ path can be established as $P\left[X_{n(n-1)(n-2) \cdots 6}, C\right]+(C, D)+$ $\left(D, Y_{n(n-1)(n-2) \cdots 6}\right)$, where $P\left[X_{n(n-1)(n-2) \cdots 6}, C\right]$ denotes an $X_{n(n-1)(n-2) \cdots 6}-C$ path passing all the vertices of the $K_{5}^{4}$ but $D$ and $Y_{n(n-1)(n-2) \cdots 6}$ exactly once.
In the discussion above, $X_{1}$ and $Y_{1}\left(X_{n(n-1)(n-2) \cdots 6}\right.$ and $Y_{n(n-1)(n-2) \cdots 6}$ are the entry and exit 4-vertices of $A_{1}\left(A_{n(n-1)(n-2) \cdots 6}\right)$, respectively. Additionally, we use $X_{i}$ and $Y_{i}$ to denote the entry and exit 4 -vertices of $A_{i}$, respectively, for $2 \leq i \leq n(n-1)(n-2) \cdots 6-1$. Let $L_{i}\left(Q_{i}\right)$ be the 4-vertex of $A_{i}$ that is not connected to $A_{i-1}\left(A_{i+1}\right)$. A hamiltonian $X_{i}-Y_{i}$ path in the $K_{5}^{4}$ formed by $A_{i}$ can be established according to the following four cases. Case $1 . Q_{i}=X_{i}$ and $L_{i}=$ $Y_{i}$. A hamiltonian $X_{i}-Y_{i}$ path can be established easily. Case 2. $Q_{i} \neq X_{i}$ and $L_{i}=Y_{i}$. A hamiltonian $X_{i}-Y_{i}$ path can be established as $\left(X_{i}, Q_{i}\right)+P\left[Q_{i}, Y_{i}\right]$ where $P\left[Q_{i}, Y_{i}\right]$ denotes a $Q_{i}-Y_{i}$ path passing all the vertices of the $K_{5}^{4}$ but $X_{i}$ exactly once. Case 3 . $Q_{i}=X_{i}$ and $L_{i} \neq Y_{i}$. A hamiltonian $X_{i}-Y_{i}$ path can be established as $P\left[X_{i}, L_{i}\right]+\left(L_{i}, Y_{i}\right)$, where $P\left[X_{i}, L_{i}\right]$ denotes an $X_{i}-L_{i}$ path passing all the vertices of the $K_{5}^{4}$ but $Y_{i}$ exactly once. Case $4 . Q_{i}=X_{i}$ and $L_{i} \neq Y_{i}$. If $Q_{i}=L_{i}$, a hamiltonian $X_{i}-Y_{i}$ path can be established as $\left(X_{i}, U_{i}, L_{i}, V_{i}, Y_{i}\right)$, where $U_{i}$ and $V_{i}$ are the other two 4 -vertices of $A_{i}$ than $X_{i}, Y_{i}$, and $L_{i}$. If $Q_{i} \neq L_{i}$, a hamiltonian $X_{i}-Y_{i}$ path can be established as $\left(X_{i}, Q_{i}, U_{i}, L_{i}, Y_{i}\right)$, where $U_{i}$ is the other 4-vertex of $A_{i}$ than $X_{i}, Y_{i}, L_{i}$, and $Q_{i}$.

Clearly the hamiltonian paths obtained above interleaved with 4-edges $\left(Y_{1}, X_{2}\right),\left(Y_{2}, X_{3}\right), \ldots$, $\left(Y_{n(n-1)(n-2) \cdots 6-1}, X_{n(n-1)(n-2) \cdots 6}\right)$ form a $P_{4}$. Next, we show the $P_{4}$ good. (Cond. 1) holds because $X_{1}$ is the beginning 4 -vertex and $Y_{n(n-1)(n-2) \cdots 6}$ is the ending 4-vertex. (Cond. 3) holds for the reason as follows. Recall that $w_{j}=u_{k}$ for some $k \in$ $\{2,3, \ldots, n\}-\left\{j, a_{1}, a_{2}, \ldots, a_{n-5}\right\}$. After executing a $k$-partition on the $P_{4}, X_{1}=\left\langle x_{1} x_{2} \ldots x_{n}\right\rangle_{4}$ forms a $K_{4}^{3}$. Since $x_{k}=u_{k}=w_{j}$, the beginning 3 -vertex is not connected to $W$. Similarly, the ending 3 vertex is not connected to $D$. In the following, we show (Cond. 2) holds. Let $X=\left\langle x_{1} x_{2} \ldots x_{n}\right\rangle_{4}$, $Y=\left\langle y_{1} y_{2} \ldots y_{n}\right\rangle_{4}$, and $Z=\left\langle z_{1} z_{2} \ldots z_{n}\right\rangle_{4}$ be arbitrary three consecutive 4 -vertices in the $P_{4}$. Assuming $p=\operatorname{dif}(X, Y)$ and $q=\operatorname{dif}(Y, Z)$, we show $x_{p} \neq z_{q}$ according to three cases. If $X$ is the exit 4-vertex of $A_{i}$ for some $1<i<n(n-1)(n-2) \cdots 6-1$, then $Y$ is the entry 4 -vertex of $A_{i+1}$ and $Z$ is the second 4 -vertex in the hamiltonian path established for the $K_{5}^{4}$ formed by $A_{i+1}$. Besides, $p \neq j=q$. Suppose conversely $x_{p}=z_{q}$. Then, $Z$ is not connected to $A_{i}$ (recall that the pair of 4 -vertices in $A_{i}$ and $A_{i+1}$ that are not adjacent are $\left\langle x_{1} \ldots x_{q-1} z_{p} x_{q+1} \ldots x_{n}\right\rangle_{4}$ and $\left.<z_{1} \ldots z_{q-1} x_{p} z_{q+1} \ldots z_{n}\right\rangle_{4}$, respectively, where $x_{q}=$ $z_{p} \neq x_{p}=z_{q}$ and $x_{i}=z_{i}$ for all $1 \leq i \leq n$ and $i \neq\{p, q\})$. According to our construction for the $P_{4}$, $Z$ should be the third or fourth or fifth 4 -vertex in the hamiltonian path established for the $K_{5}^{4}$ formed by $A_{i+1}$, which is a contradiction. If $Z$ is the entry 4 -vertex of $A_{i}$ for some $1<i \leq n(n-1)(n-2) \cdots 6$, then $x_{p} \neq z_{q}$ can be shown similarly. Otherwise, if $X, Y$, and $Z$ belong to the same 4 -vertex, then $p=\operatorname{dif}(X, Y)=\operatorname{dif}(X, Z)=\operatorname{dif}(Y, Z)=q$. Since $X$ and $Z$ are different 4 -vertices, we have $x_{p} \neq z_{q}$. This completes the proof. 
As with similar arguments to prove the above, we can show the following lemmas. Due to space limitation, the details are omitted.

\section{Lemma 3.6 $A$ good $P_{3}$ can be obtained from a good $P_{4}$.}

Proof: We suppose $P_{4}=\left[A_{1}, A_{2}, \ldots, A_{n(n-1)(n-2) \cdots 5}\right]$. Without loss of generality, we assume that the $P_{4}$ is obtained from $S_{n}$ by executing an $\left(a_{1}, a_{2}, \ldots, a_{n-4}\right)$ partition, where $a_{1} a_{2} \ldots a_{n-4}$ is an arrangement out of $\{2,3, \ldots, n\}$. Since the $P_{4}$ is good, there exists $j \in\{2,3, \ldots, n\}-\left\{a_{1}, a_{2}, \ldots, a_{n-4}\right\}$ so that after executing a $j$-partition on the $P_{4}$, the beginning (ending) 3-vertex in $A_{1}\left(A_{n(n-1)(n-2) \cdots 5}\right)$ is not adjacent to $A_{2}\left(A_{n(n-1)(n-2) \cdots 5-1}\right)$. Besides, each $A_{i}$ forms a $K_{4}^{3}$, where $1 \leq i \leq n(n-1)(n-2) \cdots 5$. In the rest of the proof, we construct a good $P_{3}$ from the $P_{4}$ by establishing a hamiltonian path for each $K_{4}^{3}$. Suppose $u=$ $u_{1} u_{2} \ldots u_{n}$ and $v=v_{1} v_{2} \ldots v_{n}$ are the beginning and ending vertices, respectively. We establish a hamiltonian path for the $K_{4}^{3}$ formed by $A_{1}$ as follows. Let $X_{1}$ be the beginning 3 -vertex (in $A_{1}$ ) and $W=\left\langle w_{1} w_{2} \ldots w_{n}\right\rangle_{3}$ be a 3 -vertex in $A_{1}$ which is different from $X_{1}$ and has $w_{j}=u_{k}$ for some $k \in\{2,3, \ldots, n\}-\left\{j, a_{1}, a_{2}, \ldots, a_{n-4}\right\}$. We note that $X_{1}$ is not connected to $A_{2}$. Since there are three 2 -edges between $A_{1}$ and $A_{2}$, there is another 3 -vertex $Y_{1} \notin\left\{X_{1}, W\right\}$ in $A_{1}$ which is connected to $A_{2}$. A hamiltonian $X_{1}-Y_{1}$ path can be established as $\left(X_{1}, W\right)+P\left[W, Y_{1}\right]$, where $P\left[W, Y_{1}\right]$ denotes a $W-Y_{1}$ path passing all the vertices of the $K_{4}^{3}$ but $X_{1}$ exactly once. for

Then we continue to establish a hamiltonian path

the $K_{4}^{3}$ formed by $A_{n(n-1)(n-2) \cdots 5}$. Let $Y_{n(n-1)(n-2) \cdots 5}$ be the ending 3 -vertex (in $A_{n(n-1)(n-2) \cdots 5}$ ) and $D=$ $<d_{1} d_{2} \ldots d_{n}>_{3}$ be the 3 -vertex in $A_{n(n-1)(n-2) \cdots 5}$ that is different from $Y_{n(n-1)(n-2) \cdots 5}$ and has $d_{j}=$ $v_{k}$ (here, $k$ is identical with that $k$ appearing in the situation of $\left.A_{1}\right)$. There exists a 3 -vertex $X_{n(n-1)(n-2) \cdots 5} \neq D$ in $A_{n(n-1)(n-2) \cdots 5}$ which is connected to $A_{n(n-1)(n-2) \cdots 5-1}$. A hamiltonian $X_{n(n-1)(n-2) \cdots 5}-Y_{n(n-1)(n-2) \cdots 5}$ path can be established as $P\left[X_{n(n-1)(n-2) \cdots 5}, D\right]+\left(D, Y_{n(n-1)(n-2) \cdots 5}\right)$, where $\quad P\left[X_{n(n-1)(n-2) \cdots 5}, D\right]$ denotes an $X_{n(n-1)(n-2) \cdots 5}-D$ path passing all the vertices of the $K_{4}^{3}$ but $Y_{n(n-1)(n-2) \cdots 5}$ exactly once. In the discussion above, $X_{1}$ and $Y_{1}\left(X_{n(n-1)(n-2) \cdots 5}\right.$ and $\left.Y_{n(n-1)(n-2) \cdots 5}\right)$ are the entry and exit 3-vertices of $A_{1}$ $\left(A_{n(n-1)(n-2) \cdots 5}\right)$, respectively. By $X_{i}$ and $Y_{i}$ we denote the entry and exit 3 -vertices of $A_{i}$, respectively, for $2<i \leq n(n-1)(n-2) \cdots 5-1$. Let $L_{i}\left(Q_{i}\right)$ be the 3 -vertex in $A_{i}$ that is not connected to $A_{i-1}\left(A_{i+1}\right)$. A hamiltonian $X_{i}-Y_{i}$ path for the formed by $A_{i}$ can be established according to the following four cases.

Case 1. $Q_{i}=X_{i}$ and $L_{i}=Y_{i}$. A hamiltonian $X_{i}-Y_{i}$ path can be established easily.

Case 2. $Q_{i} \neq X_{i}$ and $L_{i}=Y_{i}$. A hamiltonian $X_{i}-Y_{i}$ path can be established as $\left(X_{i}, Q_{i}\right)+P\left[Q_{i}, Y_{i}\right]$, where $P\left[Q_{i}, Y_{i}\right]$ denotes a $Q_{i}-Y_{i}$ path passing all the vertices of the $K_{4}^{3}$ but $X_{i}$ exactly once.

Case 3. $Q_{i}=X_{i}$ and $L_{i} \neq Y_{i}$. A hamiltonian $X_{i}-Y_{i}$ path can be established as $P\left[X_{i}, L_{i}\right]+\left(L_{i}, Y_{i}\right)$, where $P\left[X_{i}, L_{i}\right]$ denotes an $X_{i}-L_{i}$ path passing all the vertices of the $K_{4}^{3}$ but $Y_{i}$ exactly once.

Case 4. $Q_{i} \neq X_{i}$ and $L_{i} \neq Y_{i}$. Since the $P_{4}$ is good, Lemma 3.1 assures that each 3 -vertex of $A_{i}$ is connected to $A_{i-1}$ or $A_{i+1}$. Hence, $Q_{i} \neq$ $L_{i}$. A hamiltonian $X_{i}-Y_{i}$ path can be established as $\left(X_{i}, Q_{i}, L_{i}, Y_{i}\right)$. The hamiltonian paths obtained above interleaved with: 3-edges $\left(Y_{1}, X_{2}\right),\left(Y_{2}, X_{3}\right), \ldots$, $\left(Y_{n(n-1)(n-2) \cdots 5-1}, X_{n(n-1)(n-2) \cdots 5}\right)$ form a $P_{3}$. Moreover, the $P_{3}$ is good, with the same arguments as the proof of Lemma 3.5.

Q.E.D.

\section{Lemma 3.7 There is a good $P_{3}$ in $S_{5}$.}

Proof: Suppose $u=u_{1} u_{2} u_{3} u_{4} u_{5}$ and $v=v_{1} v_{2} v_{3} v_{4} v_{5}$ are the beginning and ending vertices, respectively. We assume $u_{i} \neq v_{i}$ for $i \in\left\{a_{1}, a_{2}, \ldots, a_{k}\right\} \subseteq$ $\{1,2,3,4,5\}$ and $u_{i}=v_{i}$ otherwise, where $2 \leq k \leq \overline{5}$ and $a_{1}<a_{2}<\cdots<a_{k}$. First, an $a_{k}$-partition is executed on $S_{5}$, and so a $K_{5}^{4}$ results. We use $U_{4}$ and $V_{4}$ to denote the beginning and ending 4-vertices, respectively. In the following, we construct a good $P_{3}$ according to the values of $k$.

Case 1. $k=2$. We assume $a_{1} \neq 1$. The discussion for $a_{1}=1$ is very similar. For ease of explanation, we assurne, without loss of generality, $a_{1}=2$ and $a_{2}=3$. We then arbitrarily select $l=4$ from the set $\{2,3,4,5\}-\left\{a_{1}, a_{2}\right\}=\{4,5\}$, and let $\left.S=\left\langle s_{1} s_{2} s_{3} s_{4} s_{5}\right\rangle_{4}=<* * s_{3} * *\right\rangle_{4}$ be the vertex of the $K_{5}^{4}$ with $\left(s_{a_{2}}=\right) s_{3}=u_{4}\left(=u_{l}\right)$. Since there are five vertices in the $K_{5}^{4}$, we can find a 4-vertex $Z=\left\langle z_{1} z_{2} z_{3} z_{4} z_{5}\right\rangle_{4}=\left\langle * * z_{3} * *\right\rangle_{4} \notin\left\{U_{4}, S, V_{4}\right\}$ with $\left(z_{a_{2}}=\right) z_{3} \neq v_{1}$. Let $T$ be the other vertex than $U_{4}, S, Z$, and $V_{4}$ in the $K_{5}^{4}$. A hamiltonian path for the $K_{5}^{4}$ can be established as $\left(U_{4}, S, T, Z, V_{4}\right)$, which constitutes a $P_{4}=\left[U_{4}, S, T, Z, V_{4}\right]$. An $l$-partition is then executed on the $P_{4}$, and so each 4 -vertex of the $P_{4}$ forms a $K_{4}^{3}$. By establishing a hamiltonian path for each $K_{4}^{3}$, a good $P_{3}$ can be obtained as follows.

First we establish a hamiltonian path for the $K_{4}^{3}$ formed by $V_{4}$. Let $V_{3}=\left\langle * * v_{3} v_{4} *\right\rangle_{3}$ be the ending 3 -vertex (in $V_{4}$ ) and $D=\left\langle d_{1} d_{2} d_{3} d_{4} d_{5}\right\rangle_{3}=$ $\left.<* * v_{3} d_{4} *\right\rangle_{3}$ be the 3 -vertex of $V_{4}$ that is not connected to $Z$. Since $\left.s_{a_{2}}=u_{l}=v_{l}=\right) v_{4} \neq z_{3}\left(=z_{a_{2}}\right)$, $V_{3}$ is connected to $Z$. So, $D \neq V_{3}$. Moreover, since there are three 3 -edges between $Z$ and $V_{4}$, there exists a 3 -vertex $X \neq V_{3}$ in $V_{4}$ which is connected to $Z$. A hamiltonian path for the $K_{4}^{\mathbf{3}}$ can be established as $P[X, D]+\left(D, V_{3}\right)$, where $P[X, D]$ denotes an $X-D$ path passing all the vertices of the $K_{4}^{3}$ but $V_{3}$ exactly once.

We then continue to establish a hamiltonian path for the $K_{4}^{3}$ formed by $U_{4}$. We have $d_{l}=v_{r}$ for some $r \in\{2,3,4,5\}-\left\{a_{2}, l\right\}=\{2,5\}$. We note $r \neq 1$ because $D$ is the 3 -vertex in $V_{4}$ that is not connected to $Z$ (which implies $d_{l}=z_{a_{2}} \neq v_{1}$ ). Let $U_{3}=\left\langle * * u_{3} u_{4} *\right\rangle_{3}$ be the beginning 3-vertex (in 
$\left.U_{4}\right)$ and $W=\left\langle w_{1} w_{2} w_{3} w_{4} w_{5}>_{3}=<* * u_{3} w_{4} *>_{3}\right.$ be the 3 -vertex in $U_{4}$ that is different from $U_{3}$ and has $\left(w_{l}=\right) w_{4}=u_{r}$. We note that $U_{3}$ is not connected to $S$ because $\left(s_{a_{2}}=\right) s_{3}=u_{4}\left(=u_{l}\right)$. So, there exists another 3 -vertex $Y \notin\left\{U_{3}, W\right\}$ in $U_{4}$ which is connected to $S$. A hamiltonian path for the $K_{4}^{3}$ can be established as $\left(U_{3}, W\right)+P[W, Y]$, where $P[W, Y]$ denotes a $W-Y$ path passing all the vertices of the $K_{4}^{3}$ but $U_{3}$ exactly once.

Since there are three 3 -edges between every two adjacent 4-vertices of the $P_{4}$, distinct entry and exit 3vertices can be determined for $S, T$, and $Z$. Then, a hamiltonian path from the entry 3 -vertex to the exit 3 vertex can be established for each $K_{4}^{3}$ formed by them, similar to the proof of Lemma 3.6 , in order to satisfy (Cond. 2). The obtained hamiltonian paths interleaved with used 3 -edges form a $P_{3}=\left[A_{1}, A_{2}, \ldots, A_{20}\right]$, where $A_{1}=U_{3}, A_{2}=W, A_{19}=D$, and $A_{20}=V_{3}$. In the following we show that the $P_{3}$ is good.

Clearly, (Cond. 1) holds, and with the same arguments as the proof of Lemma 3.5, (Cond 2) also holds. After executing an $r$-partition on the $P_{3}$, each $A_{i}$ forms a $K_{3}^{2}$, where $1 \leq i \leq 20$. Without loss of generality, we assume $r=\overline{2}$. Let $U_{2}=\left\langle * u_{2} u_{3} u_{4} *\right\rangle_{2}$ (in $A_{1}$ ) and $V_{2}=\left\langle * v_{2} v_{3} v_{4} *\right\rangle_{2}$ (in $A_{20}$ ) be the beginning and ending 2 -vertices, respectively. Since $\left(u_{r}=\right) u_{2}=w_{4}\left(=w_{l}=w_{\text {dif }\left(A_{1}, A_{2}\right)}\right), U_{2}$ is not connected to $W=A_{2}$. Similarly, since $\left(v_{r}=\right) v_{2}=d_{4}(=$ $\left.d_{l}=d_{\text {dif }\left(\boldsymbol{A}_{19}, \boldsymbol{A}_{20}\right)}\right), V_{2}$ is not connected to $D=A_{19}$. Thus, (Cond. 3 ) holds.

Case 2. $k=3$. The method for constructing a good $P_{3}$ is almost the same as Case 1 , but $k$ is changed to 3 and $l$ is selected from the set $\{2,3,4,5\}-\left\{a_{1}, a_{2}, a_{3}\right\}$.

Case 3. $k=4$. We assume $u_{l}=v_{l}$, where $l \in\{1,2,3,4,5\}-\left\{a_{1}, a_{2}, a_{3}, a_{4}\right\}$. If $u_{t} \neq v_{t}, u_{t} \neq$ $v_{a_{4}}$, and $v_{t} \neq u_{a_{4}}$ for some $t \in\left\{a_{1}, a_{2}, a_{3}\right\}-\{1\}$, then two 4 -vertices $Q=\left\langle q_{1} q_{2} q_{3} q_{4} q_{5}\right\rangle_{4}$ and $H=$ $<h_{1} h_{2} h_{3} h_{4} h_{5}>_{4}$ with $q_{a_{4}}=u_{t}$ and $h_{a_{4}}=v_{t}$ are determined. A hamiltonian path for the $K_{5}^{4}$ can be established as $\left(U_{4}, Q, T, H, V_{4}\right)$, where $T$ is the other 4 -vertex than $U_{4}, Q, H$, and $V_{4}$. The hamiltonian path forms a good $P_{4}=\left[U_{4}, Q, T, H, V_{4}\right]$ for the following reasons. (Cond. 1) and (Cond 2) hold with the same reasons as Case 1. (Cond. 3) holds as a consequence of executing a $t$-partition on the $P_{4}$. By Lemma 3.6, a good $P_{3}$ can be obtained from the $P_{4}$.

Otherwise, if there exists no $t \in\left\{a_{1}, a_{2}, a_{3}\right\}-\{1\}$ satisfying $u_{t} \neq v_{t}, u_{t} \neq v_{a_{4}}$, and $v_{t} \neq u_{a_{4}}$, then $a_{1}=1$, which implies $l \neq 1$. The method for constructing a good $P_{3}$ is almost the same as Case 1 , but $k$ is changed to 4 and $l$ is unique.

Case $4 . \quad k=5$. There exists a number $t \in$ $\left\{a_{1}, a_{2}, a_{3}, a_{4}\right\}-\{1\}$ satisfying $u_{t} \neq v_{t}, u_{t} \neq v_{a_{5}}$, and $v_{t} \neq u_{a_{5}}$. A good $P_{3}$ can be obtained similar to Case 3 .

We note that $S_{3}$ forms a cycle of length six. The following two lemmas have been shown in [4].

Lemma 3.8 [4] Suppose $X$ and $Y$ are two adjacent 3-vertices in a $P_{3}$, and let $\left(c_{0}, c_{1}, \ldots, c_{5}\right)$ denote the cycle formed by $X$. Then, the vertices of $X$ that are connected to $Y$ are $c_{j}$ and $c_{(j+3) \bmod 6}$ for some $0 \leq j \leq$
5.

Lemma 3.9 [4] Suppose $X=\left\langle x_{1} x_{2} \ldots x_{n}\right\rangle_{3}, Y=$ $\left\langle y_{1} y_{2} \ldots y_{n}\right\rangle_{3}$, and $Z=\left\langle z_{1} z_{2} \ldots z_{n}\right\rangle_{3}$ are arbitrary three consecutive 3 -vertices in a $P_{3}$. If $x_{\text {dif }(X, Y) \neq} \neq$

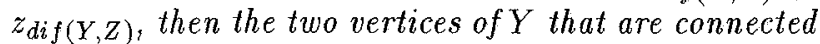
to $X$ are disjoint from the two of $Y$ that are connected to $Z$.

Lemma 3.10 Suppose $u$ and $v$ are arbitrary two distinct vertices of $S_{n}$ with $n>4$. A longest $u-v$ path can be constructed from a good $P_{3}$. The longest path has length $n !-1$ if dist $(u, v)$ is odd, and $n !-2$ if $\operatorname{dist}(u, v)$ is even, where dist $(u, v)$ is the distance between $u$ and $v$.

Proof: It is not difficult to check that this lemma holds for $S_{4}$ (recall that $S_{n}$ is vertex symmetric). Hence, we assume $n \geq 5$. According to Lemmas?? and 3.7 , a good $P_{3}=\left[A_{1}, A_{2}, \ldots, A_{n(n-1)(n-2) \cdots 4}\right]$ can be obtained in $S_{n}$. We use $\left(c_{i, 0}, c_{i, 1}, \ldots, c_{i, 5}\right)$ to denote the cycle formed by $A_{i}$, where $1<i \leq$ $n(n-1)(n-2) \cdots 4$. According to Lemma 3.8, two vertices $c_{1, j}$ and $c_{1,(j+3) \bmod 6}\left(c_{n(n-1)(n-2) \cdots 4, k}\right.$ and

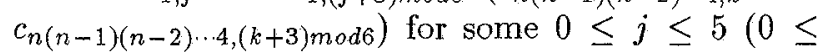
$k \leq 5)$ are connected to $A_{2}\left(A_{n(n-1)(n-2) \cdots 4-1}\right)$. We have $u \neq\left\{c_{1, j}, c_{1,(j+3) \bmod 6}\right\}$, for otherwise the beginning 2 -vertex must be connected to $A_{2}$, which contradicts (Cond. 3). Similarly, $v \neq$ $\left\{c_{n(n-1)(n-2) \cdots 4, k}, c_{n(n-1)(n-2) \cdots 4,(k+3) \bmod 6}\right\} . \quad$ Since $A_{1}\left(A_{n(n-1)(n-2) \cdots 4}\right)$ forms a cycle of length $6, u(v)$ is adjacent to $c_{1, j}$ or $c_{1,(j+3) \bmod 6}\left(c_{n(n-1)(n-2) \cdots 4, k}\right.$ or

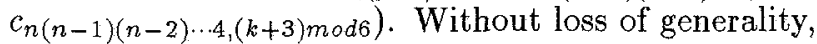
we assume $u$ is adjacent to $c_{1, j}$. We let $x_{1}=u$ and $y_{1}=c_{1, j}$, and select $x_{i}$ and $y_{i}$, sequentially, for $i=$ $2,3, \ldots, n(n-1)(n-2) \cdots 4-1$ from each $A_{i}$ so that $x_{i}$ is adjacent to both $y_{i-1}$ and $y_{i}$, and $y_{n(n-1)(n-2) \cdots 4-1}$ is connected to $A_{n(n-1)(n-2) \cdots 4}$. Lemmas 3.8 and 3.9 assure the existence of $x_{i}$ and $y_{i}$. Since $A_{1}$ contains a hamiltonian $u-y_{1}$ path and each $A_{i}$ contains a hamiltonian $x_{i}-y_{i}$ path, a hamiltonian $u-y_{n(n-1)(n-2) \cdots 4-1}$ path (of length $n !-6)$ for $S_{n}-\left\{A_{n(n-1)(n-2) \cdots 4}\right\}$ thus results.

Next we augment the $u-y_{n(n-1)(n-2) \cdots 4-1}$ path with a longest $y_{n(n-1)(n-2) \cdots 4-1}-v$ path. Without loss of generality, we assume $y_{n(n-1)(n-2) \cdots 4-1}$ is adjacent to $c_{n(n-1)(n-2) \cdots 4, k}$. If dist $(u, v)$ is odd, any $u-v$ path has odd length because $S_{n b}$ is bipartite. So, $v \neq$

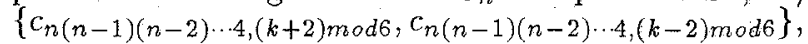
for otherwise there exists a $u-v$ path of even length, which is a contradiction. Since we also have $v \neq$ $\left\{c_{n(n-1)(n-2) \cdots 4, k}, c_{n(n-1)(n-2) \cdots 4,(k+3) \bmod 6}\right\}, v$ should be $c_{n(n-1)(n-2) \cdots 4,(k+1) \bmod 6}$

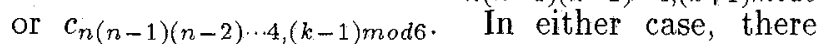
exists a hamiltonian $c_{n(n-1)(n-2) \cdots 4, k}-v$ path (of length 5) for $A_{n(n-1)(n-2) \cdots 4}$. Similarly, if $\operatorname{dist}(u, v)$ is even, $v$ should be $c_{n(n-1)(n-2) \cdots 4,(k+2) \bmod 6}$ or

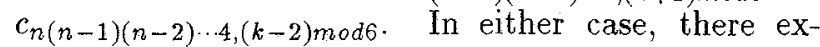


ists a $c_{n(n-1)(n-2) \cdots 4, k}-v$ path of length 4 in $A_{n(n-1)(n-2) \cdots 4}$. This completes the proof. Q.E.D.

The following theorem holds as an immediate consequence of Lemma 3.10 .

Theorem $3.11 S_{n}$ with $n \geq 4$ is strongly hamiltonian-laceable.

\section{Concluding remarks}

In this paper we have introduced the concept of strongly hamiltonian-laceability for star graphs. By extanding our results, we can show that the $n$ dimensional star graph, where $n \geq 6$, remains strongly hamiltonian-laceable, even if $n-\overline{4}$ random edge faults happen, and show that the $n$-dimensional star graph, where $n \geq 6$, remains strongly hamiltonian-laceable, even if $n-3$ random edge faults happen, exclusive of two exceptions in which there are at most two vertices missing from the longest paths.

\section{References}

[1] S. B. Akers, D. Harel and B. Krishnamurthy, "The star graph: an attractive alternative to the $n$ cube," Proceedings of the International Conference on Parallel Processing, 1987, pp. 393-400.

[2] S. B. Akers, B. Krishnamurthy, "A group-theoretic model for symmetric interconnection networks," IEEE Transactions on Computers, vol. 38, no. 4 , pp.555-566, 1989.

[3] J. S. Jwo, S. Lakshmivarahan, and S. K. Dhall, "Embedding of cycles and grids in star graphs," Journal of Circuits, Systems, and Computers, vol. 1, no. 1, pp.43-74, 1991.

[4] Y. C. Tseng, S. H. Chang, and J. P. Sheu, "Faulttolerant ring embedding in star graphs," Proceedings of the International Parallel Processing Symposium, 1996, pp. 660-665.

[5] S. A. Wong, "Hamiltonian cycles and paths in butterfly graphs," Networks, vol. 26, pp. 145-150, 1995. 